\title{
Acute Appendicitis: Evidence Based Management
}

\author{
Medina Andrade Luis Angel MD ${ }^{1}$, Misael Silva Gonzalez $\mathrm{MD}^{2}$, StephannyVanestty \\ Woolf Telléz $\mathrm{MD}^{3}$, Palomo Pineda Omar Marin $\mathrm{MD}^{3}$,Stephanie Serrano Collazos MD4, \\ Jose Daen Jonathan Cabañas Gomez $\mathrm{MD}^{4}$, Adrian Fernandez Vega Torres $\mathrm{MD}^{5}$, \\ Jessica González Noriega MD ${ }^{6}$, Arsenio Torres Delgado $\mathrm{MD}^{7}$, González León \\ Fernando David MD ${ }^{7}$
${ }^{1}$ General Surgery Department, General Regional Hospital \#30, IMSS, México. Corresponding author. Plutarco Elias Calles, México City
${ }^{2}$ General Surgery Department. Ixtapaluca High Specialty Regional Hospital, México.
${ }^{3}$ General Surgery Department, General Regional Hospital \#17, IMSS, Mexico.
${ }^{4}$ General Surgery Department, Ecatepec Las Americas General Hospital, Ecatepec de Morelos, México.
${ }^{5}$ General Surgery Department, National Medical Center XXI Century, IMSS, México.
${ }^{6}$ General Surgery Department. General Regional Hospital \#1, IMSS, México.
${ }^{7}$ General Surgery Departmen, Tulancingo General Hospital, Hidalgo, México.

\begin{abstract}
Although is the most frequent surgical emergency around the world, is unbelievable how many different management options could be found between different hospital centers, and inclusive in the same hospital by different surgeons, many of them ignoring actual research results, perpetuating obsolete practices without evidence based background.

The objective of this article is to confirm and resume the current better evidence based management in acute appendicitis, to create more uniformed management and improve the results of this so common pathology.

Material and Methods: A review of the literature was performed using the words "appendicitis", "appendectomy", "appendicitis management" and "appendicitis diagnosis". Searching results were limited to Cochrane studies initially by their high quality systematic reviews about this topic, and some other recent articles were included to complement this review in case some information were not found in the Cochrane library.

Results: Background and physical examination by a surgeon is enough for diagnosis. In child and elderly patient ultrasound could be very useful and the gold standard for diagnosis is CT scan. Antibiotic prophylaxis must be administered in all cases, an if it results in an uncomplicated appendicitis, suspended after surgery. Drains are not indicated, including complicated cases. Irrigation with solution or antibiotics like Imipenem diluted is associated with diminished infection rates. Wound closure could be achieved safely by a subcuticular stitch, with absorbable suture. Medical treatment for appendicitis is associated with high failure rates and higher cost of attention. Laparoscopic approach is safe and must be performed if available.
\end{abstract}

Conclusion: Evidence based management in appendicitis could provide the greatest benefit to patients, leading to better results with fewer complications and increased patient satisfaction.

Keywords: Acute Appendicitis, Appendectomy, Laparoscopic appendectomy, open appendectomy, appendicitis complications.

\section{INTRODUCTION}

Appendicitis is the most frequent surgical emergency in the world, only after caesarean, and it is presented in 6 to $10 \%$ of population along live, less frequent in childhood and elderly.

The diagnosis has evolved along time with the improvement in imaging studies like ultrasound and, actually, CT scan as the more sensitive and specific, but not always needed to confirm diagnosis.

Antibiotic progression in use and indications allow surgeons to treat patients with the better combinations of this at the precise time to diminish doses, time of administration and increase patients wellbeing with less hospital stay and complications associated with the procedure.

Although is the most frequent surgical emergency around the world, is unbelievable how many different management options could be found between different hospital centers, and inclusive in the 
same hospital by different surgeons, many of them ignoring actual research results, perpetuating obsolete practices without evidence based background.

The objective of this article is to confirm and resume the current better evidence based management in acute appendicitis, to create more uniformed management and improve the results of this so frequent pathology.

\section{Material AND MethodS}

A review of the literature was performed using the words "appendicitis", "appendectomy", "appendicitis management" and "appendicitis diagnosis". Searching results were limited to Cochrane studies initially by the high quality systematic reviews about this topic. After initial search 6 articles were identified but after two different surgeons evaluate each of this, only 5 were included for this review purpose. An additional search was carried out in Pubmed and UptoDate, about related recent research in appendicitis surgical treatment, with initial inclusion of 12 studies but, after analysis by two different surgeons, only 4 were included, and 4 more that were cited in this were used as evidence by their high quality and relevance.

\section{RESUltS}

\subsection{Diagnosis}

Appendicitis diagnosis have evolved along time with CT scan as the actual gold standard for this purpose. The diagnostic accuracy of an experienced surgeon in the majority of cases is enough and the use of imaging methods would be only in selective cases. This is the case of pediatric patients where appendicitis diagnosis must be difficult according to younger age, difficult physical exam and lack of symptoms expressions by younger child. For the previous reasons is indicated the use of ultrasonography in this population to assess better accuracy and preferably in pediatric centers with skilled sonographic personnel. Current guidelines recommend the use of CT scan only in cases of complex appendicitis, where the ultrasound was not helpful or in elderly patients secondary to the many ways of presentation in this population without classical appendicitis signs or clinical history (Intestinal obstruction, diffuse pain, anorexia, feeding intolerance).

\subsection{Antibiotics}

With all the improvements in surgery technique and perioperative management in acute appendicitis, the main and more frequent complication after appendectomy is wound infection. The evolution in knowledge about antibiotic and their proper use in different scenarios allow us to establish the better moment to administer, the kind of antibiotic to use and the period to be administered after surgery according to findings.

Actual guidelines in the use of antibiotics recommend to administer a prophylactic dose for gram (-) aerobic and anaerobic, 60 minutes before surgical incision. The recommended antibiotics include a cephalosporin like cefoxitin ( 1 to $2 \mathrm{~g}$ IV), ampicillin/sulbactam ( $3 \mathrm{~g}$ IV), the combination of ceftriaxone $(50 \mathrm{mg} / \mathrm{kg}$ IV) PLUS metronidazole $(30 \mathrm{mg} / \mathrm{kg}$ IV), or in penicillin allergic patients the combination of clindamycin plus ciprofloxacin ${ }^{1-5}$.

Personally I prefer the combination of ceftriaxone plus metronidazole for the excellent results obtained in personal protocols, and this allow us to obtain superficial wound infection rates $<2 \%$ and abscess rates $<5 \%$. In cases of uncomplicated appendicitis, the recommendation is not to administer more antibiotics after surgery because it is not related with diminished complication rates. After complicated appendicitis cases antibiotics must be continued until inflammatory systemic response signs are absents ${ }^{5-7}$.

\subsection{Drains}

Drains use was the rule in cases of complicated appendicitis. As in many other surgical procedures like cholecystectomy or colon surgery, it has been confirmed that the use of drains is only associated with a longer hospital stay, estimated near $34.4 \%$ increase of average, and in the specific case of appendicitis is associated with an increased incidence of intra-abdominal abscess compared with no drain management, reason why this practice must be avoided ${ }^{8,9}$. 


\subsection{Irrigation}

Other measure that has been confirmed to diminish wound infection is the irrigation of wound before skin closure. In a clinical trial by Parcells JP. and his group, the use of wound irrigation with $1 \mathrm{~g}$ imipenem diluted in saline solution before skin closure was associated with diminished wound infection rate $(0.5 \%)$, followed by saline solution irrigation with $7.3 \%$ of wound infection rate and finally Dakin's solution with $15 \%{ }^{10}$.

\subsection{Wound Closure}

Primary wound closure was outlaw in the past in cases of complicated appendicitis arguing the high risk of wound infection and that after some days washing, it would be closed by second intention. Many years ago was confirmed that primary closure is a safe technique that does not increase the complication associated like superficial infection or abscess formation, with less morbidity for patient and better aesthetic results ${ }^{11}$. In studies by Khajouei H. et al. and Francis Serour et al. in different population groups, wound closure technique with absorbable intradermic stitch and the classic technique with non-absorbable were compared, resulting in an equivalent complication rate and better cosmetic results from the first, showing the feasibility and safe of this technique.

Other practice that have been confirmed to be safe is the appendectomy skin closure with subcuticular stitches of an absorbable suture, with polyglactin 910 as the most used with excellent results, similar infection and abscess rates compared with non-absorbable stitches in some trials and inclusive with better results in others, confirming that the skin closure with separate non absorbable stitches represents an increased relative risk of complications of 2.91 compared to a continuous intradermal stitch $^{6}$.

\subsection{Antibiotics Vs Surgery}

Multiple clinical trials have been developed to evaluate the use of antibiotics as non-complicated appendicitis treatment. This trials have many factors that doesn`t allow this practice as a feasible one, because at first, CT scan and the analysis by a radiologist must be mandatory, resources not always available in all hospitals and during the night, to confirm the non-complicated appendicitis. For this reasons we have the limited resource in must medical centers and that all patients with suspected appendicitis would be confirmed by CT scan previous to begin the management with antibiotics. With antibiotic treatment and interval appendectomy it has been confirmed that almost $30 \%$ of patients present failure with progression of symptoms or relapse in the first three weeks after discharge, with complication rates of $20 \%$ in other trials, representing a high risk for patients and higher total medical attention cost over surgery ${ }^{6,12}$.For this reason medical management have been only recommended in cases of non-complicated appendicitis confirmed by CT scan and in medical centers with full time surgeon to evaluate patients frequently during the first days of management for surgical intervention if needed, and always communicating the potential risk of this therapy to patient and parents.

\subsection{Laparoscopic Vs Open}

Finally, laparoscopic approach has been introduced in almost all surgical procedures, and multiple studies have been carried out to confirm the superiority over open approach and the case of appendectomy is not the exception. Laparoscopic approach has the advantage of better results in obese patients, employed ones or females, shorter hospital stay, less post-operative pain and better aesthetic results. This technique is associated with higher incidence of intra-abdominal abscess, higher cost, the need for laparoscopic skills by surgeon and equipment available in all shifts. Evidence based guidelines recommend the use of laparoscopy in patients with suspected appendicitis in those clinical settings where surgical expertise and equipment are available and affordable, especially in female, obese or employed patients unless it is contraindicated ${ }^{5,13}$. Clinical evidence is not conclusive and can't recommend the superiority of single incision laparoscopic appendectomy over classical laparoscopic approach ${ }^{14}$.

\section{CONClusion}

During surgical formation many of the surgical techniques or management guidelines evolve along time and sometimes surgeons are reluctant to change, perpetuating handlings that not always provide the greatest benefit to patients. It is our responsibility to stay up to date and remain open to change, to give patients the best known management schemes, leading to better results with fewer complications and increased patient satisfaction. 


\section{REFERENCES}

[1] Andersen BR, Kallehave FL, Andersen HK.Antibiotics versus placebo for prevention of postoperative infection after appendicectomy...Cochrane Database of Systematic Reviews 2005, Issue 3. Art. No.: CD001439.DOI: 10.1002/14651858.CD001439.pub2.

[2] Ortega Gezzer (2012) An evaluation of surgical site infections by wound classification system using the ACS-NSQIP. J Surg Res 174:33-38.

[3] Andersen BR et al (2001) Review: antibiotics reduce wound infections and intra-abdominal abscesses in patients having appendectomy. Cochrane Database Syst Rev [cited 28 Sep 2014]. Available in: http://ebn.bmj.com/content/5/2/49.full.pdf

[4] Ein SH, Sandler A (2006) Wound infection prophylaxis in pediatric acute appendicitis-a 26-year prospective study. J PediatrSurg 41:538-541.

[5] Smink Douglas, I. Sobel David, Weiser Martin and Chen Weinlang. Management of acute appendicitis. From www.uptodate.com 2016. http://www.facmed.unam.mx/bmnd/dirijo _gbc.php?bib_vv=22

[6] Medina Andrade L.A., Perez Muñoz F.Y., Jimenez Baez M.V. and Serrano Collazos S. Appendectomy Skin Closure Technique, Randomized Controlled Trial: Changing Paradigms (ASC). World Journal of Surgery 2016

[7] Daskalakis K., Juhlin C. and Påhlman L. THE USE OF PRE- OR POSTOPERATIVE ANTIBIOTICS IN SURGERY FOR APPENDICITIS: A SYSTEMATIC REVIEW. Scandinavian Journal of Surgery 0: 1-7, 2013.

[8] Cheng Y, Zhou S, Zhou R, Lu J, Wu S, Xiong X, Ye H, Lin Y, Wu T, Cheng N. Abdominal drainage to prevent intra-peritoneal abscess after open appendectomy for complicated appendicitis. Cochrane Database of Systematic Reviews 2015, Issue 2. Art. No.: CD010168. DOI: 10.1002/14651858.CD010168.pub2.

[9] Tander B et al (2003) The utility of peritoneal drains in children with uncomplicated perforated appendicitis. PediatrSurgInt 19(7):548-550

[10] Parcells JP, Mileski JP, Gnagy FT, Haragan AF et al (2006) Using antimicrobial solution for irrigation in appendicitis to lower surgical site infection rates. Am J Surg 198(6):875-880.

[11] Chau GY (2012) Can delayed primary wound closure decrease incidence of wound infection after appendectomy in patients with perforated appendicitis? J Chin Med Assoc 75:249-250.

[12] Wilms IMHA, de Hoog DENM, de Visser DC, Janzing HMJ. Appendectomy versus antibiotic treatment for acute appendicitis.Cochrane Database of Systematic Reviews 2011, Issue 11. Art. No.: CD008359. DOI: 10.1002 / 14651858.CD008359.pub2.

[13] Sauerland S, Jaschinski T, Neugebauer EAM. Laparoscopic versus open surgery for suspected appendicitis. Cochrane Database of Systematic Reviews 2010, Issue 10. Art. No.: CD001546. DOI: 10.1002/ 14651858.CD001546.pub3.

[14] Rehman H, Rao AM, Ahmed I.Single incision versus conventional multi-incision appendicectomy for suspected appendicitis. Cochrane Database of Systematic Reviews 2011, Issue 7. Art. No.: CD009022. DOI: 10.1002/14651858.CD009022.pub2. 